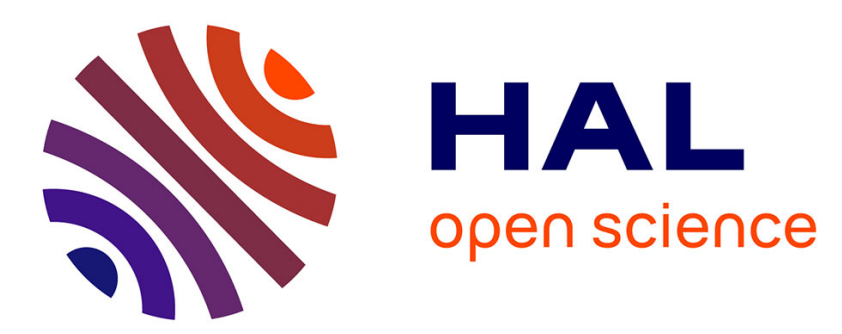

\title{
A Simple and Efficient Non-Model Based Cable Tension Control
}

\author{
João Cavalcanti Santos, Marc Gouttefarde
}

\section{To cite this version:}

João Cavalcanti Santos, Marc Gouttefarde. A Simple and Efficient Non-Model Based Cable Tension Control. CableCon 2021 - 5th International Conference on Cable-Driven Parallel Robots, Jul 2021, Virtual, France. pp.297-308, 10.1007/978-3-030-75789-2_24 . lirmm-03217093

\section{HAL Id: lirmm-03217093 https://hal-lirmm.ccsd.cnrs.fr/lirmm-03217093}

Submitted on 4 May 2021

HAL is a multi-disciplinary open access archive for the deposit and dissemination of scientific research documents, whether they are published or not. The documents may come from teaching and research institutions in France or abroad, or from public or private research centers.
L'archive ouverte pluridisciplinaire HAL, est destinée au dépôt et à la diffusion de documents scientifiques de niveau recherche, publiés ou non, émanant des établissements d'enseignement et de recherche français ou étrangers, des laboratoires publics ou privés. 


\title{
A Simple and Efficient Non-Model Based Cable Tension Control *
}

\author{
João Cavalcanti Santos ${ }^{1}$ and Marc Gouttefarde ${ }^{1}$ \\ ${ }^{1}$ LIRMM, Univ Montpellier, CNRS, France \\ \{cavalcanti, gouttefarde\}@lirmm.fr
}

\begin{abstract}
Although the control of cable tensions plays a paramount role in the control of Cable-Driven Parallel Robots (CDPRs), studies addressing the subject are very few. The present paper proposes and experimentally validates a simple cable tension feedback control scheme. In order to avoid issues related to friction and saturation of the feedback correction, a velocity-controlled winch is considered. The closed-loop scheme consists of an ordinary PI applied with respect to the measured cable tensions. Thanks to its simplicity, the main advantage of the proposed scheme is its ease of implementation. The experimental validation is performed using two scenarios. First, a simplified case consisting of a single degree-of-freedom CDPR is considered. Finally, the performance is validated with a a six degrees-of-freedom CDPR fully-constrained by eight cables.
\end{abstract}

Keywords: Cable tension control $\cdot$ Non-model based $\cdot$ Velocity-controlled motor.

\section{Introduction}

Most often, motion control schemes of Cable-Driven Parallel Robots (CDPRs) define, at some point, a set of desired cable tensions. Therefore, a strategy should be defined to convert desired cable tensions to a variable that is meaningful to the motor drivers, typically motor torques (or currents), positions or velocities. Such control strategies are referred to as cable tension control schemes.

The majority of published experimental results were obtained with open-loop cable tension control. A common solution is the application of motor torques compensating the friction in the winches 27/15 which led to satisfying results in the context of position tracking control [6] and of force-based control [12]. Nevertheless, the application of a closed-loop approach should notably improve the robustness to the uncertainties present in the controlled system.

A closed-loop cable tension control can be seen as a simplified case of feedback force control which is a well established topic in robotics [16 17. Its variants, which can be exemplified by impedance control [3] and hybrid position/force

\footnotetext{
* This work was supported by the European Union's H2020 Program (H2020/20142020) under the grant agreement No. 732513 (HEPHAESTUS project).
} 
control [1], were successfully implemented in both serial [10] and parallel robots 8]. Nevertheless, studies proposing closed-loop cable tension control strategies in the context of CDPR control are few.

Based on an elastic cable model, Kraus et al. propose a cable tension control scheme using motor positions as control inputs to the motor drivers [5]. This work presents details on the identification of the dynamic system and experimental results on position tracking control. Similar methods are used in [4] and in [11. Nevertheless, the application of a position-controlled inner-loop may lead to the saturation of the feedback correction. Kraus et al. proposes the implementation of an anti-windup strategy in order to overcome this issue.

Besides, torque-controlled winches allow the motor torques to be directly used as the control inputs. An advanced technique was tested through numerical simulations in 9] using $\mathcal{H}_{\infty}$ robust control in order to compensate the influence of the cable sagging and elasticity. However, torque-based strategies requires a precise model of the friction present in the winches. This may be a significant issue when gear trains with a large reduction ratio are used.

The present paper proposes a rather simple control strategy considering velocity-controlled winches. This non-model based approach relies on a PI feedback correction and, therefore, presents simple implementation and tuning. Furthermore, the proposed scheme does not demand any identification of the controlled system. The control scheme can be seen as an application of a standard velocity-based admittance control, as discussed in [13. Experimental validation is fulfilled using two scenarios. First, a simplified case of a one degree-of-freedom (DoF) CDPR is considered. Finally, the application on the CDPR motion control is validated on a 6 -DoF CDPR fully-constrained by eight cables. In contrast to position-based control schemes, real-time experiments notably indicate that the proposed control scheme is not prone to saturation issues.

\section{Set-up Description}

A simple 1-DoF CDPR is first considered in order to study the cable tension control of CDPRs. This set-up is illustrated in Figure1. It consists of two cables of a CDPR connected one to each other at point $P$. The influence of gravity is neglected under the assumption that the cables are kept sufficiently taut. Accordingly, the position of the point $P$ is determined by means of the scalar $\ell$ as shown in Figure 1. For this 1-DoF CDPR, the stretched cable length, i.e. the actual length of the cable under tension, is equal to $\ell$ plus a constant value, the latter corresponding to the stretched cable length when point $P$ is in its reference position.

Cable tensions are measured with the aid of two load pins positioned in the pulley axes (as shown in Figure 1). The cable tension measurements corresponding to each cable are denoted as $\tau_{1}$ and $\tau_{2}$. Initially, neglecting the pulleys inertia and friction, it is assumed that $\tau_{1}=\tau_{2}=\tau$. Consequences of this assumption are discussed in Section 4 . 


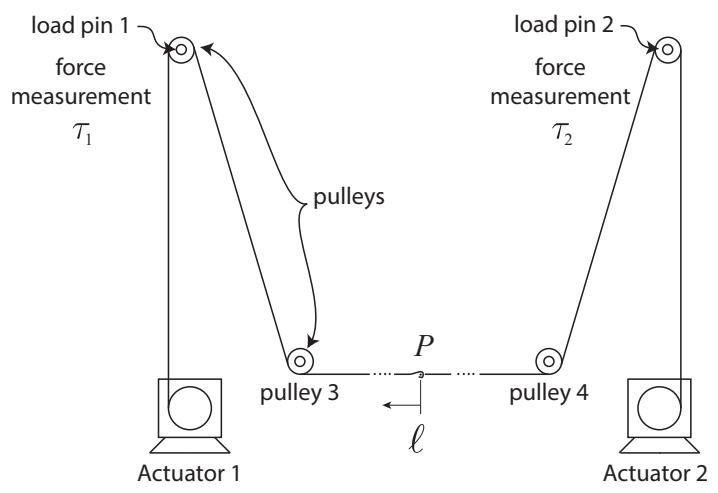

Fig. 1. An illustrative schematic view of the experimental set-up.

The synchronized movement of actuators 1 and 2 enables the control of the tension $\tau$ and the position $\ell$. As a matter of fact, the essential aspects involved in the control of the cable tensions of a CDPR can be addressed with this system. Section 3 proposes a velocity-based feedback policy able to track a desired cable tension $\tau_{d}$ by means of controlling the velocity of winch 1 , denoted as $\dot{q}$.

\section{Cable Tension Control Scheme}

The present section proposes a control scheme able to track a set of desired cable tensions by means of controlling the motor velocities. At first glance, a velocity-based strategy may seem a peculiar choice. A scheme controlling directly the motor torques may instead be considered as an appropriate option. Disregarding cable distributed mass, friction in the winches and in the routing pulleys, and dynamics forces, the torque applied by the drum is proportional to the cable tension. Moreover, independently of the chosen strategy, the innermost control loop regulates the motor current which is closely related to the motor torque.

Nevertheless, the torque applied on the drum is often transmitted from the corresponding motor by means of a gearbox which generally possesses nonnegligible friction. As a result, the torque applied on the drum and the one generated by the motor are commonly related by a highly non-linear system. This issue is demonstrated in Figure 2. The data displayed in this chart was obtained for a constant position $\ell$ of the 1-DoF CDPR shown Figure 1 whose winches have gear trains with reduction ratio of 25 - other technical details on the mechanical parts used in the experimental setup are described in the next section. One may note that a continuous variation of the motor torque results in significant discontinuities in the variation of the cable tension. This is due to the so-called stick-slip phenomenon.

In contrast, the relation between motor positions and cable tensions is much more tractable since it is mainly governed by the cable elasticity. Performing a 


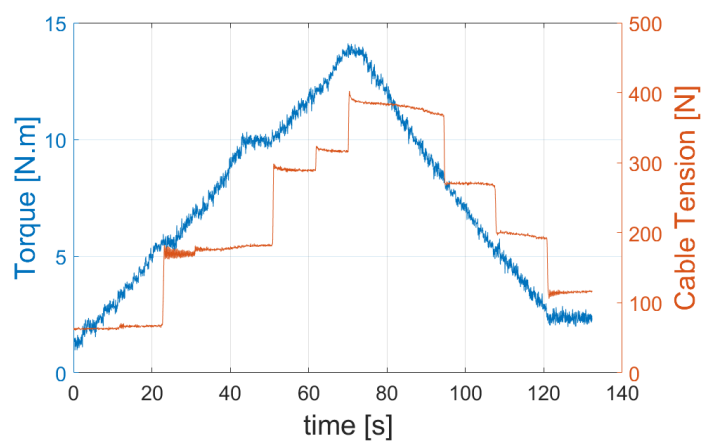

Fig. 2. Simultaneous plot of the applied torque and measured cable tension (with the cable attached to a fixed support).

test similar to the above mentioned experiment, the data depicted in Figure 3 is obtained. In this case, a continuous variation of the motor position leads to a continuous variation of the cable tension. As a matter of fact, the results presented in Figure 3 are rather obvious considering the elastic behavior of the cable. Since $\ell$ is constant in this experiment, the unstretched length variations presented in Figure 3 takes place with constant stretched length. The comparison between Figures 2 and 3 demonstrates that controlling the cable tension through the motor position should be more suitable than through the motor torque.

In general, a simple position control is able to perform a sufficiently precise position tracking independently of the external forces and torques. Hence, while positioning precision may be obtained independently of the cable tensions, the motor angular position may be controlled in order to generate a desired cable tension. Noting that the relation between the cable tension and the position is continuous and well behaved, good results with a position-based control scheme can be expected. This is indeed the approach proposed in [5]4 but some secondary complications come up with this method. Notably, the suppression of steady-state errors demands the application of an anti-windup feedback strategy.

For this reason, the present section proposes a scheme in which the motor velocity is taken as the control input. Similarly to a position-controlled winch, a simple velocity control is able to perform a sufficiently precise velocity tracking independently of the external forces and torques. Therefore, this approach is also able to reduce the influence of friction on the tracking of cable tensions. Furthermore, results presented in the remainder of the paper indicates that this velocity-based scheme is not prone to saturation issues.

As discussed earlier, a crucial advantage of the proposed control scheme lies in its simplicity of implementation. Indeed, as shown in Figure 4 , the proposed strategy consists of a simple PI feedback correction combined with a feedforward term proportional to the velocity $\dot{\ell}$. More precisely, the desired winch angular 


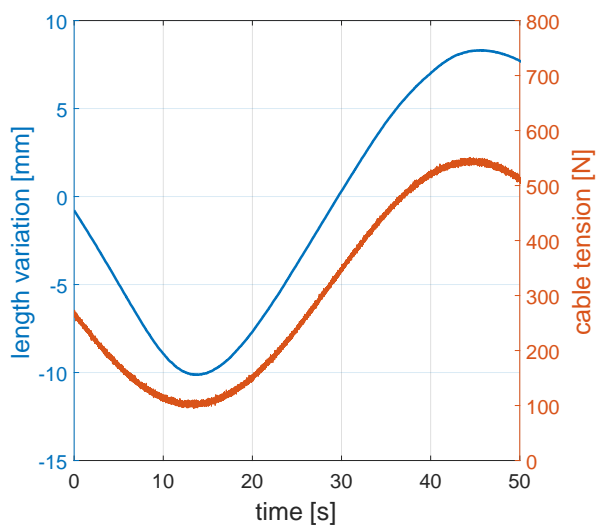

Fig. 3. Simultaneous plot of variations in the unstretched cable length and the measured cable tension (with the cable attached to a fixed support).

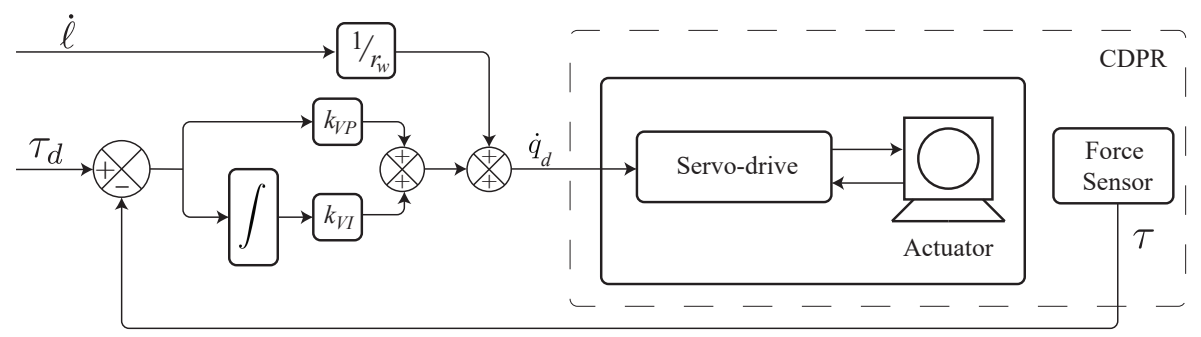

Fig. 4. Block diagram of the proposed velocity-based cable tension control scheme.

velocity $\dot{q}_{d}$ is computed according to:

$$
\dot{q}_{d}=k_{P}\left(\tau_{d}-\tau\right)+k_{I} \int_{t_{0}}^{t_{a}}\left(\tau_{d}-\tau\right) d t+\frac{\dot{\ell}}{r_{w}},
$$

with positive scalars $k_{P}$ and $k_{I}$ representing the feedback gains. The limits of integration in (1) refers to the initial and actual time, $t_{0}$ and $t_{a}$, respectively. $\tau_{d}$ is the desired cable tension and $\tau$ is the measured one. Moreover, the positive scalar $r_{w}$ denotes the radius of the winch drum. Consequently, the feedforward term $\dot{\ell} / r_{w}$ represents the motor angular velocity necessary to generate the tangential linear velocity $\dot{\ell}$ of the drum.

In practice, the value of the angular velocity $\dot{q}_{d}$ is used as a set-point in an inner-loop control responsible for tracking this velocity by means of controlling the motor currents. Typically, servo-drives are able to manage this inner-loop. While the proposed control scheme presents a remarkable simplicity, the next section shows that the resulting performance is satisfying. 


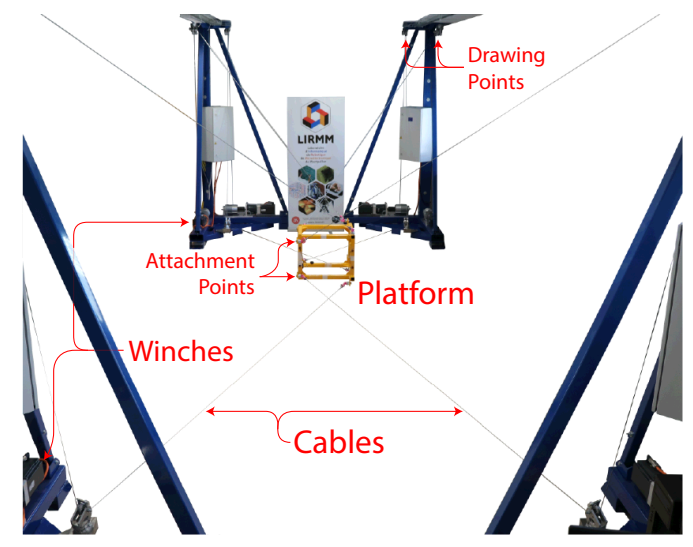

Fig. 5. HRPCable prototype.

\section{Experimental Results}

The experimental validation presented in this section was performed using the HRPCable prototype shown in Figure 5 . The mobile platform of HRPCable has six degrees of freedom and is driven by eight cables in a fully-constrained configuration. Each winch is driven by a motor Beckhoff AM8061 with nominal torque of $12.8 \mathrm{Nm}$ and nominal power of $3.46 \mathrm{~kW}$. The reduction is performed by a two stage gear train Beckhoff AG2210 with reduction ratio of 25:1. Motor servo drivers Beckhoff AX5112 are controlled by an IPC Beckhoff C6920. Control implemented in the IPC is developed in $\mathrm{C}++$ (TwinCAT environment).

Load pins Sensy 5300 1T SL positioned in the axes of the pulleys measure the cable tensions. The analogical amplifier of this sensor is integrated to the body of the load pin. Based on the authors' practical experience, this is a decisive requirement in order to obtain a reduced level of noise on the force measurements.

Section 4.1 presents the experimental validation of the control scheme proposed in Section 3. Moreover, supplementary results are discussed in Section 4.2 . The influence of the cable hysteresis and the friction in the pulleys are quantified.

\subsection{Main Validation}

First, two kinematic chains (two winches and cables) of the HRPCable prototype were used to form the 1-DoF CDPR depicted in Figure1. Figure6 shows the results obtained with the velocity-based control of Eq. (1) with a step input. The lower level is $190 \mathrm{~N}$ and the upper one is $440 \mathrm{~N}$. The rise time to reach 440 $\mathrm{N}$ is $0.4 \mathrm{~s}$. The position $\ell$ was kept constant.

Figure 7 shows the results of another test where $\tau_{d}$ varies at low frequency. This figure shows that the obtained error is negligible so that desired and measured cable tensions are virtually equivalent. Moreover, the small error obtained with a slow variation of $\tau_{d}$ shows that the proposed strategy successfully avoids the stick-slip problem. 


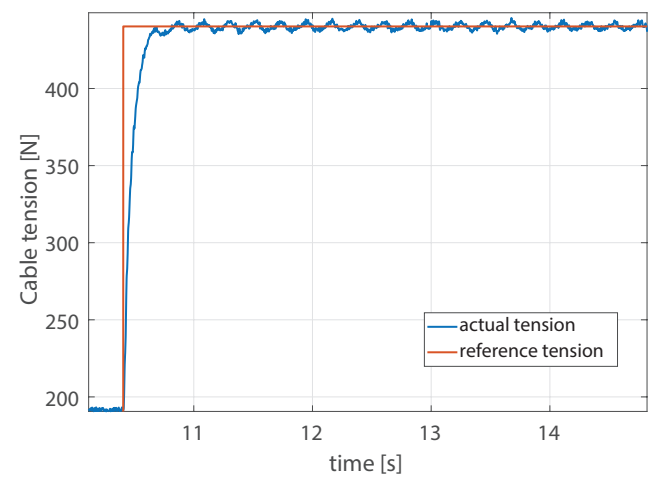

Fig. 6. Measured cable tension with a step input in the desired tension.

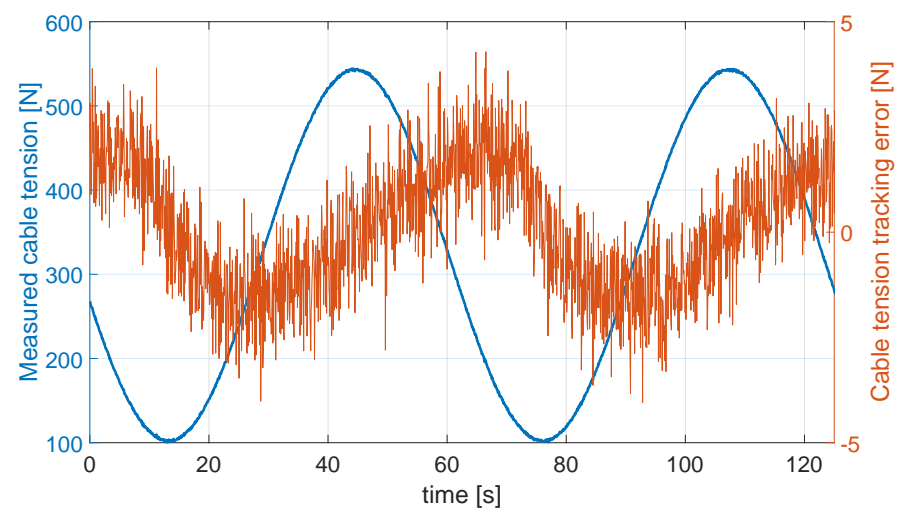

Fig. 7. Measured cable tensions and tracking errors. Displacement $\ell$ is constant and the desired cable tension is determined according $\tau_{d}=320+220 \cos (2 \pi t / 62.5)[\mathrm{N}]$. The RMS of the tracking error is equal to $1.51 \mathrm{~N}$.

Moreover, Figure 8 depicts the results for a sinusoidal $\ell$ and constant $\tau_{d}$. This figure compares strategies with and without the feedforward term $\dot{\ell} / r_{w}$. It shows that a control scheme without the feedforward term would lead to large errors when variable $\ell$ is used. The small errors obtained when the feedforward term is applied indicates that the compensation of $\dot{\ell}$ is satisfying.

Figure 9 presents the results with sinusoidal $\ell$ and $\tau_{d}$. A small delay may be observed. Nevertheless, the obtained results with the proposed control strategy are considered suitable. Accordingly, the proposed velocity-based cable tension control can be used in the CDPRs motion control.

Finally, the eight winches, cables and load pins of the HRPCable prototype were used and the proposed cable tension control scheme of Eq. (1) was implemented as part of a position tracking control strategy. More precisely, the 

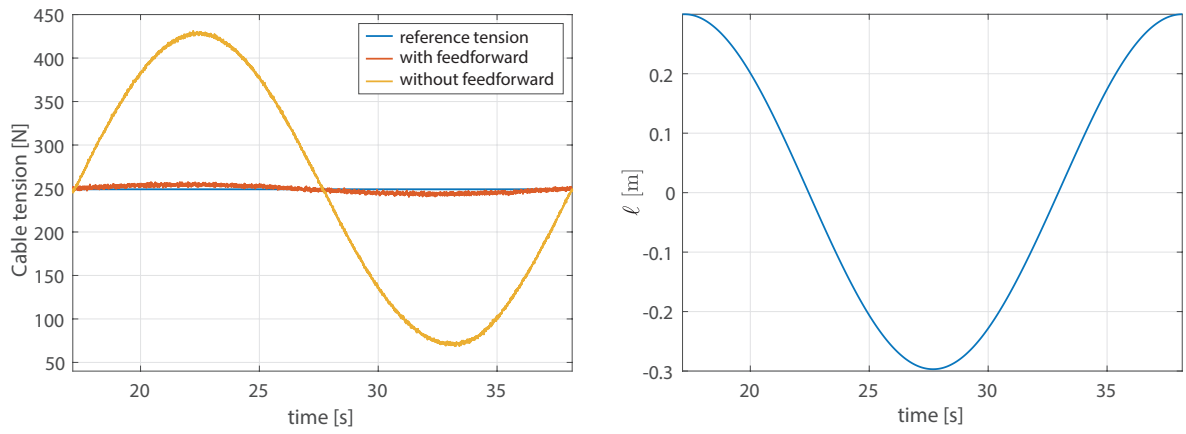

Fig. 8. Experimental results for a constant desired cable tension and a sinusoidal displacement $\ell$. The chart on the left presents the desired and measured tensions comparing schemes with and without the feedforward term $\frac{\dot{\ell}}{r_{w}}$. The chart on the right presents the sinusoidal displacement $\ell$.
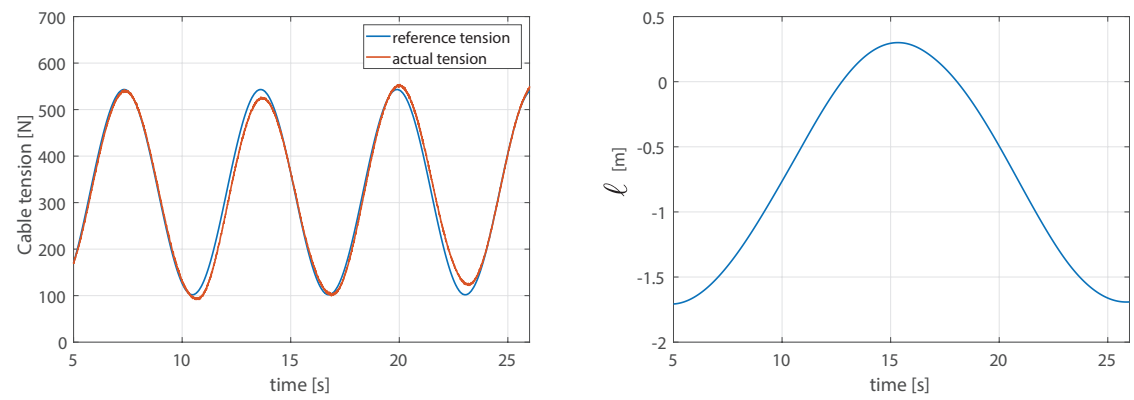

Fig. 9. Measured and desired cable tensions (on the left) and displacements $\ell$ (on the right).

Model Predictive Control (MPC) strategy proposed by the authors in [14] was implemented and used to perform the trajectory illustrated in Figure 10. The resulting measured cable tensions and cable tension tracking errors are shown in Figure 11. The tracking errors were mostly limited to $\pm 10 \mathrm{~N}$ which allows an efficient closed-loop tracking of the desired cable tensions as can be seen in Figure 11 (a).

\subsection{Additional Results}

Some additional results were obtained during the experiments described in the previous section. Figure 12 shows several repetitions of the experiment depicted in Figure 7. One can see that the relation between the cable tension and the displacement of the winch $\left(q r_{w}\right)$ is repeatable. The plot displaying the elongation vs. the cable tension indicates that the relation between stress and strain for increasing cable tensions can be reasonably approximated by an affine 


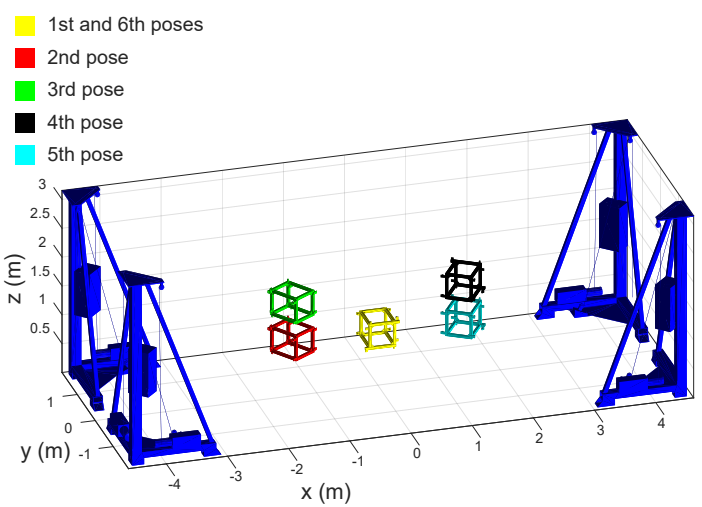

Fig. 10. Visited poses of the performed trajectory.

(a)

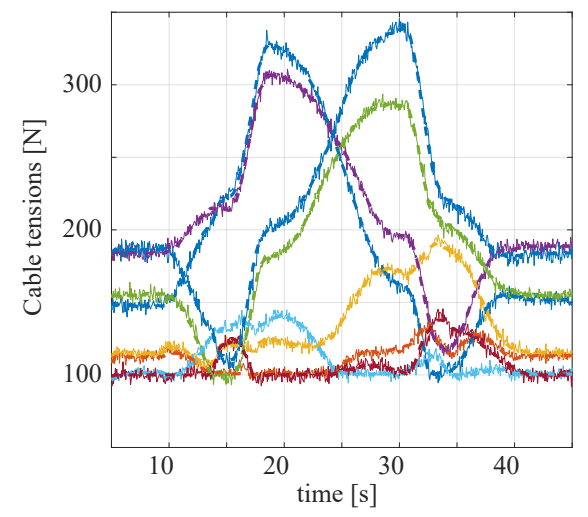

(b)

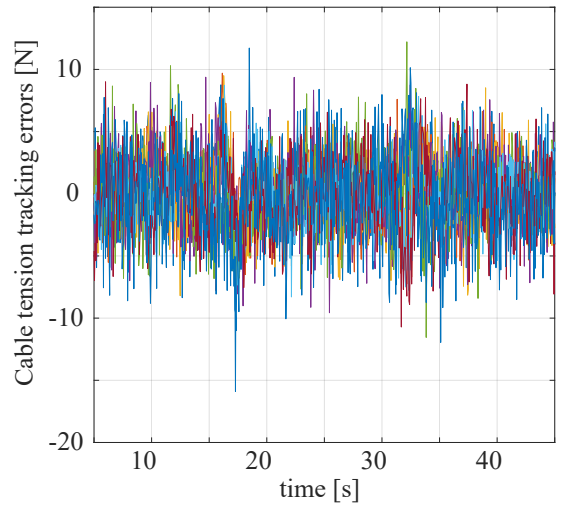

Fig. 11. (a) Graph of both measured (solid lines) and desired (dashed lines) cable tensions. (b) Tracking errors of the cable tensions.

function. Nevertheless, the values obtained for decreasing tensions shows that significant hysteresis is present.

As shown in Figure 1, there is a second load pin in pulley 2 measuring the cable tension. Figure 13 presents the values obtained with the two load pins. One may see that the cable tension $\tau_{1}$ measured by sensor 2 shifts when the direction of movement changes (velocity equals to zero). This shift is probably caused by the friction in pulleys 3 and 4 shown in Figure 1 . Since $\tau_{1}$ is the value used in the control loop, it is continuous and close to the desired cable tension.

In order to clarify the influence of the friction in the pulleys, the following simplified model $\tilde{\tau}_{2}$ of the cable tension $\tau_{2}$ can be considered:

$$
\tilde{\tau}_{2}=\tau_{1}-2 \tau_{f} \operatorname{sign}(\dot{x})+\tau_{k}
$$



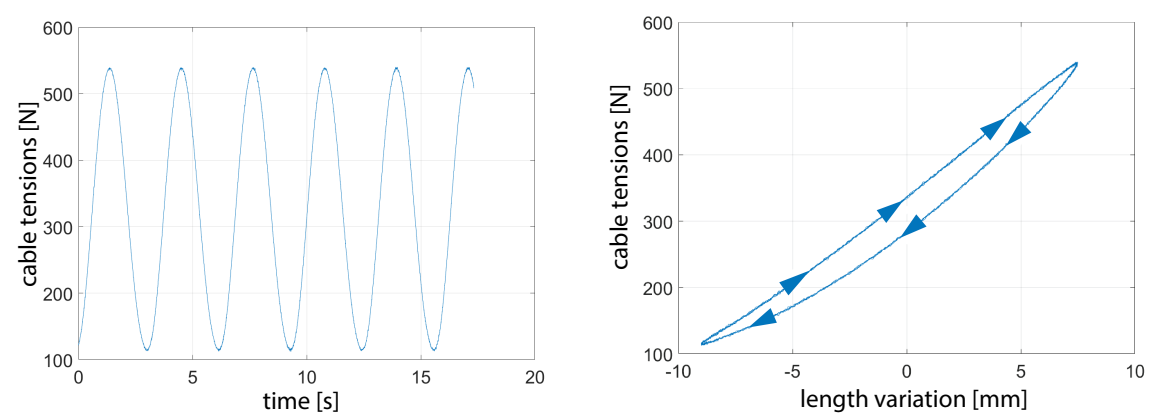

Fig. 12. Experimental results highlighting the hysteresis present in the cable elongation behavior.
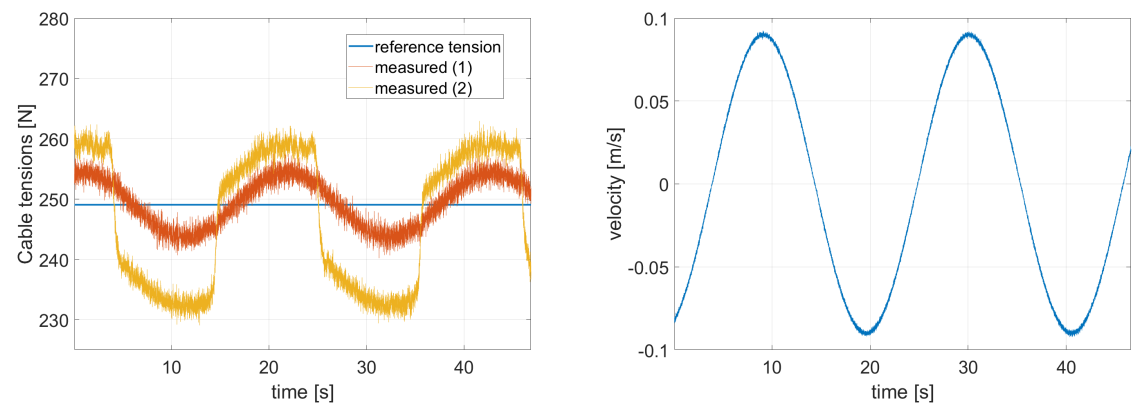

Fig. 13. Difference between cable tensions measured by load pins 1 and $2\left(\tau_{1}\right.$ and $\left.\tau_{2}\right)$.

where $\tau_{f}$ is the the dry friction force due to pulleys 3 and 4 , the friction forces in these pulleys being considered equal. The scalar $\tau_{k}$ is a constant that depends on the initialization of the system. Parameters $\tau_{f}$ and $\tau_{k}$ were identified using data from Figure 13. The fitted data is shown in Figure 14 where it can be seen that the simple model in Eq. (2) is accurate. The pulley dry friction force obtained is $\tau_{f}=3.9 \mathrm{~N}$.

\section{Conclusions}

This paper proposed and validated experimentally a non-model based cable tension control scheme. The desired cable tension is tracked by means of a velocity-controlled winch. The control scheme consists of an ordinary PI feedback correction combined with a feedforward term of the motor angular velocity. The advantage of the proposed approach in comparison to existing state-of-theart strategies is its simplicity of implementation allied to satisfying performance. This approach did not led to saturation and proved to be barely sensitive to the friction in the winch. Future works may address the influence of friction in the pulleys and the cable elasticity (and hysteresis). 


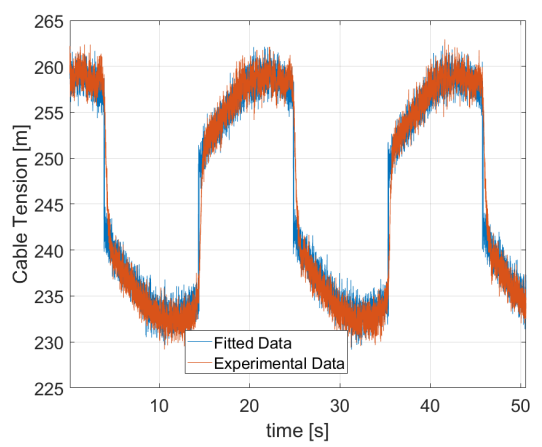

Fig. 14. Identification of the pulley friction: Measured cable tension $\tau_{2}$ (experimental data) and predicted value $\tilde{\tau}_{2}$ given by Eq. (2) (fitted data).

\section{References}

1. Chiaverini, S., Sciavicco, L.: The parallel approach to force/position control of robotic manipulators. IEEE Transactions on Robotics and Automation 9(4), 361373 (1993). https://doi.org/10.1109/70.246048

2. Fang, S., Franitza, D., Torlo, M., Bekes, F., Hiller, M.: Motion control of a tendonbased parallel manipulator using optimal tension distribution. IEEE/ASME Transactions on Mechatronics 9(3), 561-568 (2004)

3. Hogan, N.: Impedance Control: An Approach to Manipulation: Part I-Theory. Journal of Dynamic Systems, Measurement, and Control 107(1), 1-7 (mar 1985). https://doi.org/10.1115/1.3140702, https://doi.org/10.1115/1.3140702

4. Kraus, W., Miermeister, P., Schmidt, V., Pott, A.: Hybrid Position/Force Control of a Cable-Driven Parallel Robot with Experimental Evaluation. In: Flores, P., Viadero, F. (eds.) New Trends in Mechanism and Machine Science. pp. 553-561. Springer International Publishing, Cham (2015)

5. Kraus, W., Schmidt, V., Rajendra, P., Pott, A.: System identification and cable force control for a cable-driven parallel robot with industrial servo drives. In: Proceedings - IEEE International Conference on Robotics and Automation. pp. 5921-5926. IEEE (2014)

6. Lamaury, J., Gouttefarde, M.: Control of a Large Redundantly Actuated CableSuspended Parallel Robot. In: IEEE International Conference on Robotics and Automation. p. 211. IEEE (2013)

7. Lamaury, J., Gouttefarde, M., Chemori, A., Herve, P.E.: Dual-space adaptive control of redundantly actuated cable-driven parallel robots. In: IEEE International Conference on Intelligent Robots and Systems. pp. 4879-4886. IEEE (2013)

8. Merlet, J..: Force-feedback control of parallel manipulators. In: Proceedings. 1988 IEEE International Conference on Robotics and Automation. pp. 1484-1489 vol.3 (1988). https://doi.org/10.1109/ROBOT.1988.12277

9. Meunier, G., Boulet, B., Nahon, M.: Control of an overactuated cable-driven parallel mechanism for a radio telescope application. IEEE Transactions on Control Systems Technology 17(5), 1043-1054 (2009)

10. Ott, C., Mukherjee, R., Nakamura, Y.: Unified Impedance and Admittance Control. In: 2010 IEEE International Conference on Robotics and Automation. pp. 554-561 (2010). https://doi.org/10.1109/ROBOT.2010.5509861 
11. Picard, E.: Modeling and Robust Control of Cable-Driven Parallel Robots for Industrial Applications. Phd thesis, École centrale de Nantes (Dec 2019), https: //tel.archives-ouvertes.fr/tel-02954030

12. Reichert, C., Muller, K., Bruckmann, T.: Robust internal force-based impedance control for cable-driven parallel robots. In: Mechanisms and Machine Science, vol. 32, pp. 131-143. Springer, Cham (2015), http://link.springer.com/10. 1007/978-3-319-09489-2\{_\}10

13. Roy, J., Whitcomb, L.L.: Adaptive force control of position/velocity controlled robots: theory and experiment. IEEE Transactions on Robotics and Automation 18(2), 121-137 (April 2002). https://doi.org/10.1109/TRA.2002.999642

14. Santos, J.C., Chemori, A., Gouttefarde, M.: Redundancy Resolution integrated Model Predictive Control of CDPRs : Concept, Implementation and Experiments. In: Proceedings - IEEE International Conference on Robotics and Automation. pp. 3889-3895. Paris, France (2020)

15. Shang, W., Zhang, B., Zhang, B., Zhang, F., Cong, S.: Synchronization Control in the Cable Space for Cable-Driven Parallel Robots. IEEE Transactions on Industrial Electronics 66(6), 4544-4554 (2019)

16. Siciliano, B., Villani, L.: Robot Force Control. Springer US, 1 edn. (1999)

17. Whitney, D.E.: Historical perspective and state of the art in robot force control. The International Journal of Robotics Research 6(1), 3-14 (1987). https://doi.org/10.1177/027836498700600101 